\title{
Testosterone administration in women increases the size of their peripersonal space
}

Catherine Masson ${ }^{1}$, Donné van der Westhuizen ${ }^{1}$, Jean-Paul Noel $^{2}$, Adala Prevost ${ }^{1}$, Jack van Honk $^{1,3}$, Aikaterini Fotopoulou ${ }^{4}$, Mark Solms ${ }^{1}$ and Andrea Serino ${ }^{5}$

${ }^{1}$ University of Cape Town, Cape Town, South Africa

${ }^{2}$ Center for Neural Science, New York Univeristy, New York, USA

${ }^{3}$ Utrecht University, Utrecht, The Netherlands

${ }^{4}$ University College London, London, United Kingdom

${ }^{5}$ MySpace Lab, Department of Clinical Neuroscience, Center Hospitalier Universitaire

Vaudois (CHUV), University of Lausanne, Lausanne, Switzerland

\begin{abstract}
Peripersonal space (PPS) is the space immediately surrounding the body, conceptualised as a sensory-motor interface between body and environment. PPS size differs between individuals and contexts, with intrapersonal traits and states, as well as social factors having a determining role on the size of PPS. Testosterone plays an important role in regulating social-motivational behaviour and is known to enhance dominance motivation in an implicit and unconscious manner. We investigated whether the dominance-enhancing effects of testosterone reflect as changes in the representation of PPS in a within-subjects testosterone administration study in women ( $\mathrm{N}=19)$. Participants performed a visuo-tactile integration task in a mixed-reality setup. Results indicated that the administration of testosterone caused a significant enlargement of participants' PPS, suggesting that testosterone caused participants to implicitly appropriate a larger space as their own. These findings suggest that the dominance-enhancing effects of testosterone reflect at the level of sensory-motor processing in PPS.
\end{abstract}

Keywords: Visuo-tactile, hormones, embodied cognition, multisensory integration, social dominance 


\section{Introduction}

A growing body of research has demonstrated a wide range of behavioral outcomes associated with testosterone, both pro- and anti- social in nature. Broadly, testosterone is taken to play an important role in regulating social-motivational behavior, particularly related to dominance (Eisenegger, Haushofer, \& Fehr, 2011) - that is, behavior in the service of gaining or maintaining social status (Terburg \& van Honk, 2013). Indeed, in both sexes throughout the mammalian species, testosterone has been linked to social dominance and high status on a range of dominance indices (Eisenegger et al., 2011; Mazur \& Booth, 1998; Stanton \& Schultheiss, 2009; van der Westhuizen \& Solms, 2015). For instance, testosterone has been shown to reduce anxiety and physiological stress responses - particularly in anxiety-prone individuals (Hermans et al., 2007; Hermans, Putman, Baas, Koppeschaar, \& van Honk, 2006; van Honk, Peper, \& Schutter, 2005), to reduce submissive avoidance behavior (Enter, Spinhoven, \& Roelofs, 2014; Enter, Terburg, Harrewijn, Spinhoven, \& Roelofs, 2016; Terburg et al., 2016) and to promote social approach and aggression toward threatening stimuli (van Honk \& Tuiten, 2001; van Honk et al., 1999; Wirth \& Schultheiss, 2007). Testosterone is also well known for its ability to reduce certain indices of empathy, such as moral reasoning (Montoya et al., 2013) and facial expression mimicry (Hermans, Putman, \& van Honk, 2006) and to promote egocentricity (Wright et al., 2012). A number of these effects are thought to arise via interaction with the dopaminergic system (Bell \& Sisk, 2013) and aromatisation to estradiol (Eisenegger et al., 2011). Moreover, several studies show that testosterone influences behavior in an automatic and implicit way (Terburg, Aarts, \& van Honk, 2012; Terburg \& van Honk, 2013; van Honk et al., 2005), pointing to the utility of an embodied cognition approach to understand some of the mechanisms by which testosterone supports social dominance. Thus while peripheral effects of testosterone on the body are well established and known to support a variety of processes that enhance physical performance, including stamina, strength, bone mass, male virility and reduced inflammation (Bianchi, 2019; Sinervo, Miles, Frankino, Klukowski, \& DeNardo, 2000; Sinnesael, Boonen, Claessens, Gielen, \& Vanderschueren, 2011; Wang et al., 2000), research has only recently started to explore the sensory-motor processes that may be affected by testosterone in mediating its effects in dominance behavior.

Within this embodied cognition framework, a recent study (van der Westhuizen, Moore, Solms, \& van Honk, 2017) found that the administration of a single dose of testosterone to healthy women increased their implicit feeling of control over goal-directed actions - namely, an 
increase in their sense of agency. This finding was taken to suggest that feelings of control and power may manifest firstly in the body, as control over the body's actions. In the present study we aimed to further explore this association between testosterone and bodily representations by indexing whether the former facilitates social dominance in part by modulating not only the perception of one's body (or control over it; van der Westhuizen et al. (2017)) but also the encoding of space immediately surrounding the body; the peripersonal space (PPS; di Pellegrino and Làdavas (2015); Graziano and Cooke (2006); Serino (2019)).

A set of neurons in the intra-parietal sulcus (Duhamel, Colby, \& Goldberg, 1998) and ventral pre-motor (Rizzolatti, Scandolara, Matelli, \& Gentilucci, 1981a, 1981b) regions respond both to the somatosensory stimulation on the body, and to visual or auditory stimuli near but not far from the body; that is, they encode the PPS. This spatial representation remaps plastically as a function of the individual's potential of acting in space (J. P. Noel et al., 2020; Patané et al., 2019). PPS has been shown to extend after the use of a tool (Canzoneri et al., 2013; Guterstam, Szczotka, Zeberg, \& Ehrsson, 2018; Iriki, Tanaka, \& Iwamura, 1996; Serino, Bassolino, Farne, $\&$ Ladavas, 2007), to contract after immobilization (Bassolino, Finisguerra, Canzoneri, Serino, \& Pozzo, 2015) and to blur after sensory deprivation (J.-P. Noel, H.-D. Park, et al., 2018). PPS remaps in the direction of approaching movements (Brozzoli, Cardinali, Pavani, \& Farnè, 2010; J.-P. Noel et al., 2015) and as a function of the velocity of approaching stimuli, i.e., it extends towards faster stimuli as to anticipate potential contacts (Fogassi et al., 1996; J.-P. Noel, Blanke, Magosso, \& Serino, 2018). If electrically stimulated, regions hosting PPS neurons engender defensive behaviors such as ducking (Graziano \& Cooke, 2006). Thus, PPS representation is thought to mediate body-environment interactions. More recently, PPS magnitude has been shown to be modulated by social context, such as the valence of an interaction with a conspecific (Teneggi, Canzoneri, di Pellegrino, \& Serino, 2013) or the perceived moral quality of the conspecific (Pellencin, Paladino, Herbelin, \& Serino, 2018). Further, neurophysiological (Ishida, Nakajima, Inase, \& Murata, 2010) and psychophysical (Maister, Cardini, Zamariola, Serino, \& Tsakiris, 2015; Teramoto, 2018) studies have shown that PPS does not only index the space of the self (Noel, Pfeiffer, Blanke, \& Serino, 2015; Salomon et al., 2017), but also that of others (Makin, Holmes, \& Ehrsson, 2008). Thus, by measuring PPS during testosterone administration we can build upon prior work examining body perception after testosterone administration (van der Westhuizen et al., 2017) to include the space near one's body, as well as the space near bodies of others. This ability to study space around the self and others answers questions as to whether an increased dominance motivation, 
associated with testosterone, is reflected implicitly in the encoding of one's own body, the body of others, or both.

Testosterone is strongly involved in the regulation of social approach and the defence of social status (Terburg \& van Honk, 2013), while PPS mapping functions to regulate approaching and defensive behaviors. Furthermore, previous research found that testosterone modulated interpersonal distance, causing a significant reduction in the amount of personal distance that healthy male participants preferred from aggressive individuals (Wagels, Radke, Goerlich, Habel, \& Votinov, 2017). Following these lines of evidence, we hypothesised that raised testosterone would expand participants' PPS boundary when they faced a neutral stranger conferring a larger 'self-space'. In keeping with the egocentric effects of testosterone (van Honk et al., 2011; Wright et al., 2012), we hypothesised that changes in PPS would be specific only to the self and that there would be no changes in the encoding of PPS around a neutral stranger. Moreover, given that testosterone administration has been shown to reduce physiological stress responses more effectively in individuals prone to anxiety (Hermans et al., 2007), we hypothesised that the effects of testosterone administration would be most pronounced in anxious participants. To test these hypotheses, we assessed whether testosterone influences the encoding of PPS around the self and others, by mapping PPS as a function of different testosterone levels. This was achieved by means of testosterone and placebo administration in a double-blind within-subjects design. We also included personality measures to run further exploratory analyses on the role of individual differences in mediating the effects of testosterone on PPS.

\section{Methods}

Participants. 19 right-handed females from the University of Cape Town between the ages of 18 and 25 participated in the study. Based on self-report, participation occurred during the preovulatory stage, that is, during the first 10 days following last menstruation - the most stable period in a woman's cycle. Male participants were excluded as the reliability of the testosterone administration protocol has only been established in females (Tuiten et al., 2000), and necessary and safe doses and the times course of effects in males is not yet known. This sample size is on par with the vast majority of PPS studies (e.g., 20 in J. P. Noel et al. (2018); 18 and 19 in Stone, Kandula, Keizer, and Dijkerman (2018); Stone et al., (2019); 19 in Hobeika, Taffou, Carpentier, Warusfel, and Viaud-Delmon (2020)), while additionally requiring 
participants to all be female, within a particular stage of menstrual cycle, and to partake in multiple experimental sessions always at the same time of the day (see below). Further, women on hormonal medication were excluded to prevent potential confounding interactions. Participants had no history of neurological or psychiatric disease and no visual impairments. One participant's data was discarded due to excessive outliers in her data set, suggesting that she did not understand the task. All participants were financially reimbursed for their time (R350 - approximately \$25) and gave informed written consent to take part in the study, which was approved by the University of Cape Town (UCT) Psychology Department and the UCT Health Sciences Human Research Ethics Committee.

\section{Materials and Apparatus}

PPS measurement task. The PPS task was administered using an augmented reality (AR) head mounted display (HMD, an Oculus Rift, DK1) and RealiSM software (Reality Substitution Machine, http://nco.epfl.ch/realism) an in-house purpose-made software developed at the Laboratory of Cognitive Neuroscience at the Ecole Polytechnique Federale de Lausanne (EPFL). This software superimposed a programmed virtual approaching visual stimulus, travelling from far to near, on the participant's external world (perceived via cameras attached to the VR HMD) - creating a 'mixed-reality' setup (Serino et al., 2018). The approaching visual stimulus was a tridimensional virtual tennis ball, $6.5 \mathrm{~cm}$ in diameter, looming toward the face of the participant. The ball travelled in virtual space from far to near, approaching the participant's face at a velocity of $0.75 \mathrm{~m} / \mathrm{s}$. Participants were fitted with inhouse custom made (EPFL) vibrotactile devices, attached to their cheek using skin-sensitive plasters and activated for $35 \mathrm{~ms}$.

Physiological Materials - Testosterone and placebo solution. A single dose of $0.5 \mathrm{mg}$ of testosterone, with a hydroxypropyl- $\beta$-cyclodextrin liquid carrier, was administered sublingually. Following administration at this dosage, testosterone level is known to peak between 3 and $4.5 \mathrm{hrs}$ after being ingested (Tuiten et al., 2000) and the effects of this method of testosterone administration have been demonstrated on the physiological, psychological, social and economical level many times (Boksem et al., 2013; Bos, Terburg, \& van Honk, 2010; Bos, van Honk, Ramsey, Stein, \& Hermans, 2013; Hermans, Putman, Baas, et al., 2006; Hermans, Putman, \& van Honk, 2006; Schutter \& Honk, 2004; van Honk \& Schutter, 2007; van Honk et al., 2001). The placebo solution used is identical in appearance and taste as the 
testosterone. Vials were filled and coded by an external researcher to maintain double- blindadministration.

Questionnaires. We used the STAI-Trait inventory (STAI, Spielberg, et al., 1970) to measure participants trait anxiety given the hypothesized relation between anxiety and PPS (see Introduction and Sambo \& Iannetti, 2013). To assess specificity, we also used the Brief Affective Neuroscience Personality Scales (BANPS) to measure additional personality variables. The BANPS is based on the six primary-process subcortical brain emotion systems - namely, SEEKING, RAGE, FEAR, CARE, GRIEF and PLAY - which are known to confer motivational drives imperative to survival and are believed to be foundational for personality development (Davis \& Panksepp, 2011). Differences in the weighting of these motivational drives is believed to relate to variability in personality and the scale has revealed a strong relationship with the Five-Factor Model scale (Barrett, Robins, \& Janata, 2013).

Confederates. Our experiment involved the measurement of the PPS boundary in the face of a stranger unknown to the participants - both to study how PPS around the self changes in a social context with testosterone administration, but also to examine if the representation of PPS around the other changes. Thus, confederates were hired to perform this role. We matched participants and confederates on ethnicity and gender, to prevent potential confounding effects of a confederate from a different ethnic or gender group. For example, male confederates have been found to elicit a larger defensive PPS boundary than female confederates, especially in female participants (Iachini et al., 2016). In addition, only confederates who fell in a height range of $149-169 \mathrm{~cm}(10 \mathrm{~cm}$ below or above the average South African female height) were hired to ensure that height did not impact on perception of the confederate. Moreover, to prevent a familiarity effect on the second day of testing (which itself could influence the PPS boundary), a different confederate was used on each day of testing. To induce a degree of uniformity, confederates dressed in the same way. Finally, confederates were instructed to stand at a designated point approximately 1.6 meters in front of the participant and face her while maintaining a neutral expression. Confederates did not interact in any way with participants.

\section{Procedure}


Prior to their research visit, participants completed the STAI-Trait and BANPS questionnaires electronically. Each participant was allocated to four session slots - two per day (testosterone/placebo administration session and experimental session four hours later), on two separate days, two days apart. Participants were seen at the same time of day for each administration and experimental session, respectively, as testosterone fluctuations are known to occur according to the time of day (Wirth \& Schultheiss, 2007). Only one participant was seen at the lab at a time for all four sessions. Participants were randomly assigned (using a randomization engine - GraphPad) to either receive placebo or testosterone on the first day of testing, and the alternative substance on the second day of testing.

During the administration session, the testosterone or placebo solution was administered blind. Participants held the solution under their tongue for one minute, timed by the administrator, before swallowing it.

During the experimental session four hours later, participants were seated comfortably at a desk and outfitted with the vibro-tactile devise and the AR HMD. To measure PPS, we used a well-established a visuo-tactile interaction task (Serino, Canzoneri, Marzolla, di Pellegrino, \& Magosso, 2015; Serino, Noel, et al., 2015). Participants were informed that they will feel a vibration on their cheek, see a virtual ball approaching them and that a person unknown to them will enter the room and stand in front of them. They were also told that the virtual ball and confederate are task irrelevant, and instead asked to respond as quickly as possible to the tactile vibration by pressing a key on the keyboard. The travelling virtual ball was superimposed on the participants' real surroundings, captured by cameras on the HMD and presented during the task. Participants were also instructed to look in the direction of the confederate for the duration of the task, but not to interact with her. At this point, the confederate entered the room and stood in front of the participant, at a designated point in far space approximately 1.6 meters in front of the participant (see Figure 1). The experimental task was run and there was a pause half-way through the task where the participant was given the option of a short break if they felt they needed it.

The logic of the PPS measure is as follows: PPS neurons respond both to touch and the visual stimuli presented in the near space (Duhamel et al., 1998; Graziano \& Cooke, 2006). Thus, when visual stimuli are far, there should be no multisensory interaction. But when visual stimuli are presented near, within the receptive fields of visuo-tactile neurons, visuo-tactile 
multisensory interactions should speed reaction times to touch (e.g., Canzoneri et al., 2013; Serino et al., 2015). Thus, we present tactile stimulation while visual stimulus is at different distances, and we aim to determine the furthest distance from the body at which a visual stimulus significantly speeds up tactile processing. That is, the distance at which visuo-tactile RTs are significantly faster than RTs to unimodal tactile stimulation is a proxy for the PPS boundary (Serino, Noel, et al., 2015; Serino et al., 2018).

Each trial in the task was $2660 \mathrm{~ms}$ long and on each trial, tactile stimulation (vibration) was administered at one of five different temporal delays from the onset of the trial and the onset of the visual stimuli looming toward participants (after 2165, 1732, 1299, 866, and $433 \mathrm{~ms}$ ). Thus, tactile stimuli were presented when the virtual ball was at 5 different distance points from the participant (D1 - D5 - ranging from $37.12 \mathrm{~cm}$ to $167.03 \mathrm{~cm}$ from the participant, in $32.5 \mathrm{~cm}$ intervals, see Figure 1). Specifically, when the tactile stimulation was administered after $2165 \mathrm{~ms}$ from the start of the trial, the virtual ball was at the closest distance to the participant (D1). Conversely, when the tactile stimulation was administered $433 \mathrm{~ms}$ post trial onset, the virtual ball was at its furthest distance from the participant (D5).

We included three types of trials presented in a randomised order - tactile-only trials, visuotactile trials, and catch trials. $60.60 \%$ of the trials were experimental bimodal visuo-tactile trials, in which the tactile stimulus was delivered in combination with the approaching visual stimulus (as described above). $30.30 \%$ of trials were unimodal tactile-only trials, in which the tactile stimulus was delivered in the absence of the visual stimulus. These trials are considered baseline trials and are used to show the bimodal facilitation effect on RTs to tactile stimuli (see Analysis). Tactile-only trials are important in that they can be used to control for individual differences in RTs to tactile stimuli (see Analysis). In both the unimodal and bimodal trials, the tactile stimulus was delivered at one of the five distance points (D1-D5) in a randomised order, to prevent entrainment or expectancy effects. Lastly, $9.10 \%$ of trials were catch trials in which the approaching visual stimulus was presented and no tactile stimulus was delivered. Catch trials necessitate withholding a response and thus ensure that participants are attentive to the task. Further, they mitigate the entrainment of an automatic motor response and an expectancy effect, there tactile stimuli is more likely to occur the longer it has been since trial onset (Hobeika et al., 2020; Kandula, Van der Stoep, Hofman, \& Dijkerman, 2017). In total the task consisted of 165 trials: 20 visuo-tactile trials per distance (100 total) +10 tactile-only trials per distance $(50$ total $)+15$ catch trials. A fixation cross was presented at the beginning 
of the task and during the break and was offset once a key was pressed to begin trials. The duration of the task was approximately 11 minutes.

The administration and experimental sessions were repeated two days later, and were identical with the exception of the substance administered and the confederate present, who was swapped on the second day of testing to prevent a familiarity effect.
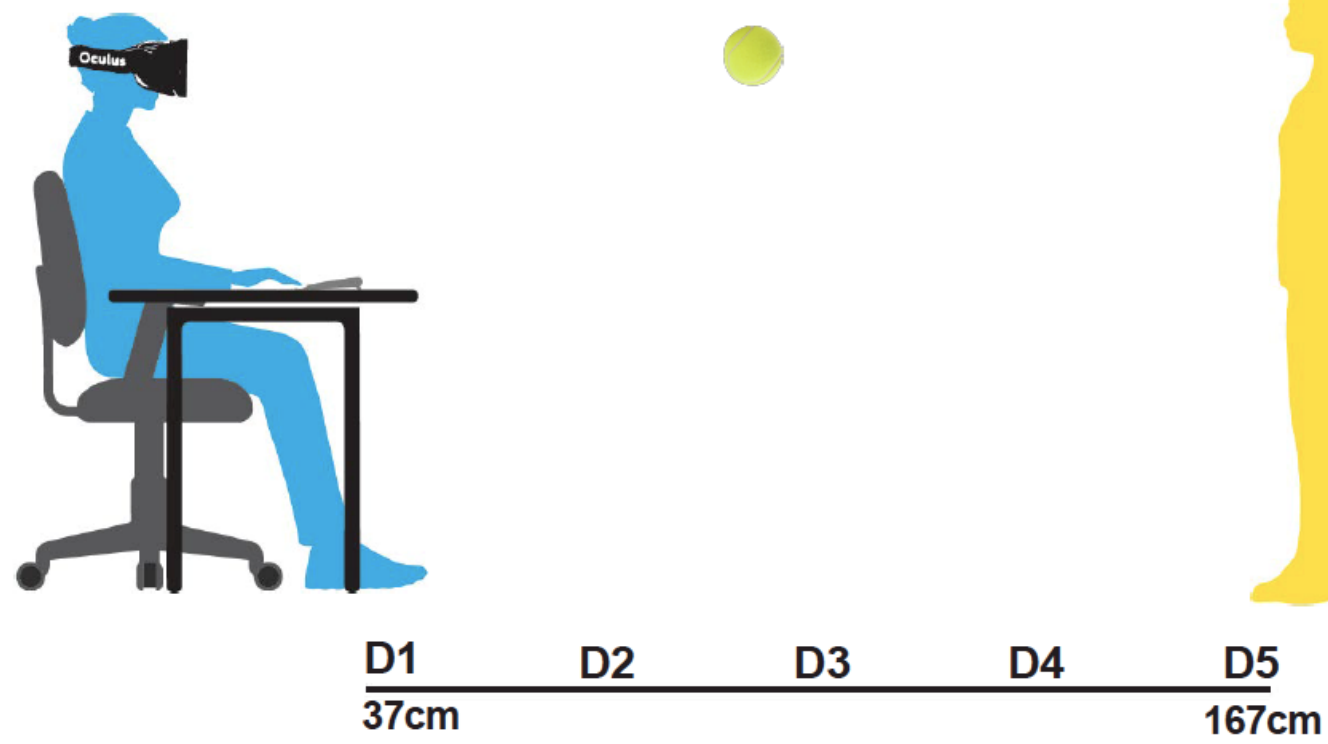

Figure 1. The experimental setup. A mixed-reality setup was used, whereby participants (blue figure) were seated at a desk and dressed with the VR HMD and a vibrotactile device on their cheek. Cameras attached to the VR HMD allowed participants to perceive their external world while also perceiving a virtual tennis ball approaching their face travelling from far to near space. Participants completed the PPS task by pressing a key on a keyboard as quickly as possible when they felt a vibration on their cheek. The task was completed while facing a confederate standing in far space (yellow figure).

\section{Analyses}

Peripersonal Space. RTs to visuo-tactile (VT) and tactile-alone (T) stimulation were recorded as the temporal duration between vibrotactile stimulus onset and button press. For each subject individually, we binned RTs as a function of the distance between the visual stimuli and the observer (D1 through D5), and as a function of sensory stimulation (VT vs. T) and testosterone condition (testosterone vs. placebo). Then, mean tactile RTs for each sensory stimulation and testosterone condition were subtracted from the analogous VT condition in order to compute "baseline-corrected" RTs (see Pfeiffer, Noel, Serino, and Blanke (2018); J.-P. Noel, H.-D. 
Park, et al. (2018), for a similar approach). This correction is employed to offset temporal expectancy effects (Kandula et al., 2017) and determine whether any putative modulation in RTs as a function of distance is truly a multisensory PPS effect (i.e., visuo-tactile RT $<$ tactile RT). That is, after correction for unisensory RTs, values under zero correspond to multisensory facilitation.

After correcting multisensory RTs in the pre-processing step described above, we first ascertained whether a PPS effect was observed in our mixed-reality setup. To do so, we computed grand average RTs to VT stimulation as a function of distance (but regardless of testosterone condition) and submitted these to a one-sample ANOVA. Planned one-sample ttests to zero were then performed in order to establish at which distances a PPS effect was observed. As detailed below, this analysis suggested a shortening of RTs when visual stimuli were presented both near the participants (i.e., self, D1) and the confederate (i.e., other, D5. See Teramoto (2018) for a similar effect). Thus, in a last step, we aimed at estimating the size and gradient of PPS representation both around the self and the confederate.

Estimation of the size and gradient of PPS was accomplished via function fitting, which permitted for fine-grain estimates (vs. solely indicating at which discrete distance did VT $\neq \mathrm{T}$ ) and served as a data-reduction technique. Visuo-tactile RTs were fit to a sigmoidal function (Eq. 1),

$$
y(x)=\frac{y_{\min }+y_{\max } \times e^{\left(x-x_{c}\right) / b}}{1+e^{\left(x-x_{c}\right) / b}}
$$

where $\mathrm{x}$ represents the distance between visual and tactile stimuli and $\mathrm{y}(\mathrm{x})$ is the RT to tactile stimulation at a given visual distance, $x . y_{\min }$ and $y_{\max }$ are saturation points of the sigmoidal, and are fixed to the slowest and fastest average RT in the VT trials. The quantities $\mathrm{x}_{\mathrm{c}}$ and $\mathrm{b}$ respectively represent the central point and a parameter dictating the slope of the sigmoidal at $\mathrm{x}_{\mathrm{c}}$, and are free to vary in order to maximize goodness of fit. The central point of this function is taken as a proxy for the size of PPS, the location of the PPS boundary, while the slope of the function (inversely proportional to $b$ ) represents the gradient with which the near (peripersonal) and far (extra-personal) space are divided (J.-P. Noel, O. Blanke, et al., 2018; J.-P. Noel, H.-D. Park, et al., 2018; Pfeiffer et al., 2018). To limit impact of the confederate on self- 
PPS estimates, distances D1 through D4 were utilized in the self condition. Similarly, distances D2 through D5 were utilized in the confederate-PPS estimates, and these were inverted (from D5 to D2) before fitting, such that distances were relative to the self (D1 through D4) or the other (D5 through D2). In this manner central point estimates for self and other were on the same scale (i.e., low values for the central point indicate a small PPS, while large values indicate a large PPS).

\section{Personality Questionnaires.}

The STAI-Trait consists of 20 questions scored on a 4-point likert scale. The BANPS consists of 33 questions scored on a 5-point likert scale. Questionnaire scores for the STAI-Trait and each of the 6 BANPS personality categories were correlated with the change in PPS size as a function of testosterone administration.

\section{Results}

\section{Peripersonal Space}

Overall, participants were very accurate at the visuo-tactile interaction task, with $0.95 \%$ omissions (i.e., lack of response to a visuo-tactile or tactile-alone trial), and $0.4 \%$ false alarms (i.e., response during a visual-only catch trial). In turn, the analysis is centered on reaction times (see Serino et al., 2015, 2017, for a similar approach).

Prior to examining the impact of testosterone on the PPS representation around the self and other (confederate), we conducted a one-way ANOVA on baseline-corrected visuo-tactile reaction times, as a function of distance but regardless of testosterone and self/other condition. This analysis revealed a significant effect of distance $\left(\mathrm{F}(4,68)=15.27, \mathrm{p}<0.001, \eta^{2}{ }_{p}=0.47\right)$, confirming that we successfully mapped a PPS effect within our mixed-reality setup. Furthermore, one-sample t-tests to zero (i.e., tactile-alone reaction times) suggested that multisensory reaction times at D1 $(\mathrm{t}(17)=4.36, \mathrm{p}<0.001)$ and $\mathrm{D} 5(\mathrm{t}(17)=2.76, \mathrm{p}=0.013)$ were significantly faster than reaction times to tactile stimulation alone, and thus seemingly a PPS representation was successfully delineated near the self (D1) and near the other (D5). Interestingly, a direct comparison between baseline-corrected multisensory reaction times at D1 (mean \pm sem; $-30 \mathrm{~ms} \pm 6.8 \mathrm{~ms}$, negative values indicating a multisensory facilitation) and 
D5 (-12ms $\pm 5.0 \mathrm{~ms})$ suggested a stronger PPS effect near the self than near another individual $(\mathrm{t}(17)=2.57, \mathrm{p}=0.020)$. Reaction times at distances D2 $-\mathrm{D} 4$ were not different from baseline (all p-values $>0.05$ ).

Having established that a multisensory PPS representation was successfully indexed (i.e., a multisensory facilitation effect that was space-dependent), we subsequently fit individual subject data and extract estimates of the location (central point) and gradient (slope) of PPS representation around the self and other, and as a function of testosterone or placebo administration (see Methods for detail). Goodness-of-fit was variable (see Serino et al. (2018)), with 4 participants showing poor fits (average $\mathrm{R}^{2}<0.2$, cut-off set a priori), and thus their data was discarded for the rest of analyses. The average $\mathrm{R}^{2}$ of the remaining participants was 0.55 .

Regarding the central point, as illustrated in Figure 2, a 2 (testosterone vs. placebo) x 2 (self vs. other) repeated-measures ANOVA revealed a significant main effect of testosterone administration $\left(\mathrm{F}(1,13)=8.9, \mathrm{p}=0.010, \eta_{p}^{2}=0.40\right)$, a significant main effect of self vs. other $\left(\mathrm{F}(1,13)=19.3, \mathrm{p}<0.001, \eta_{p}^{2}=0.59\right)$, and most importantly a significant interaction between these variables $\left(\mathrm{F}(1,13)=6.29, \mathrm{p}=0.026, \eta_{p}^{2}=0.32\right)$. The interaction was driven by the fact that the central point was farther in space for the self condition after administration of testosterone (placebo: $1.80 \pm 0.16$; testosterone: $2.42 \pm 0.35$; t $(13)=6.07, p<0.001$, Figure 2), indicating an extended PPS around one's own body. On the other hand, there was no central point difference for the other conditions, indicating that the space around the other remained unaltered by testosterone administration (placebo: $2.57 \pm 0.51$; testosterone: $2.60 \pm 0.49 ; \mathrm{t}(13)$ $=0.162, \mathrm{p}=0.87$, Figure 2).

In terms of the gradient of PPS, a 2 (testosterone vs. placebo) x 2 (self vs. other) repeatedmeasures ANOVA demonstrated a main effect of self vs. other $(F(1,13)=46.22, p<0.001$, $\left.\eta_{p}^{2}=0.78\right)$, yet no main effect of testosterone administration $(\mathrm{F}(1,13)=3.41, \mathrm{p}=0.08)$, nor an interaction between these variables $(F(1,13)=0.030, p=0.86)$. The main effect was driven by a steeper gradient around the self (b-parameter value: $0.43 \pm 0.42$ ) than around the other $(2.0 \pm 0.98$, Figure 2).

Lastly, to further support the enlargement of PPS around the self during testosterone administration we estimated the central point of the sigmoidal-like pattern of RTs via the Spearman-Karber Method (Bausenhart, Di Luca, \& Ulrich, 2018; Miller \& Ulrich, 2001). This 
approach allows for estimating psychometric parameters (e.g., central point) without performing a model fit, and thus we did not have to discard any participant. Corroborating the above finding, this analysis suggested a larger PPS around the self during testosterone administration than placebo (testosterone $=2.52 \pm 0.58 \mathrm{~ms}$; testosterone $=2.26 \pm 0.52 \mathrm{~ms} ; \mathrm{p}=$ 0.029. All other results also remain unaltered).
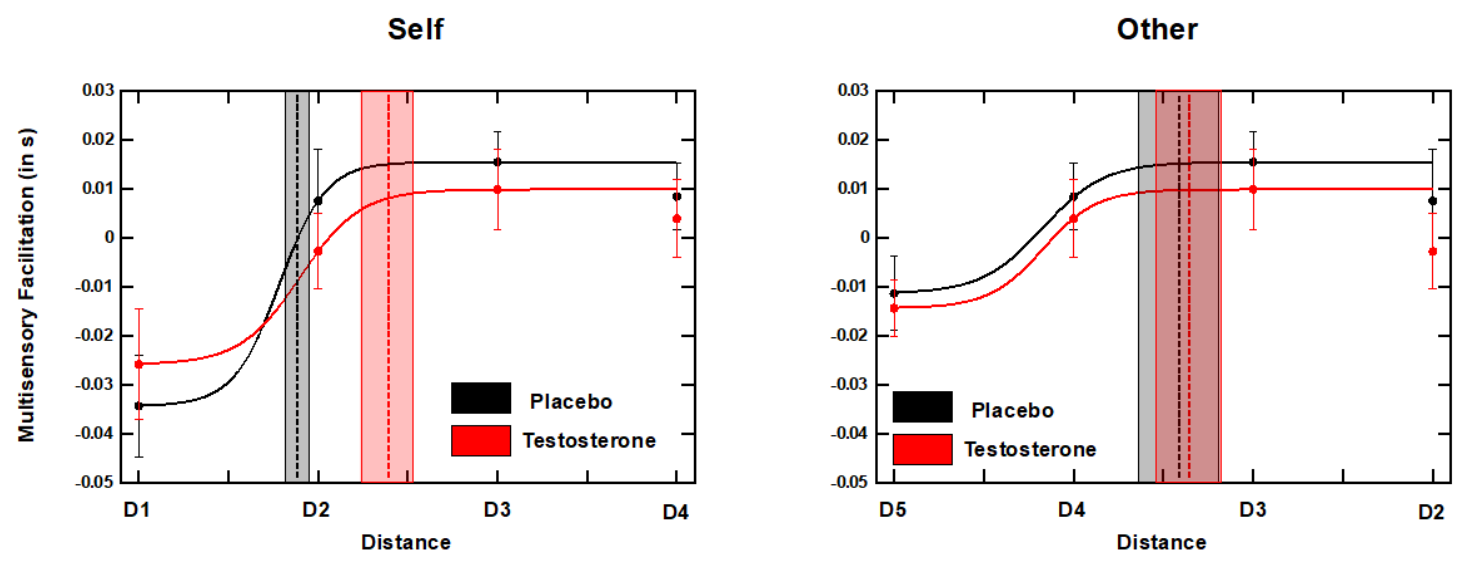

Figure 2. Effect of testosterone on PPS representation of the self and other. Left Panel; Multisensory facilitation in seconds (visuo-tactile reaction times corrected for tactile reaction times; negative values indicating multisensory facilitation) as a function of distance (near to far; 1-4) from the self, and administration of either placebo (black) or testosterone (red). Dots are mean reaction time and error bars represent $+/-1$ standard error of the mean (SEM). Dashed vertical lines represent the average central point (size) of PPS for the given condition, and shaded area around the dashed lines is SEMs. Note sigmoidal functions are fit for the average reaction time, while the vertical dashed lines are average central points of individually fitted sigmoidals. Right Panel; Multisensory facilitation as a function of distance from the other (confederate), and administration of either placebo or testosterone. Conventions follow as for the left panel.

\section{Testosterone}

Interplay Between Personality Traits and Change in Peripersonal Space Due to

Since testosterone administration seemingly enlarged the PPS representation around the self, we queried whether this remapping was related to personality variables (see J.-P. Noel, H.-D. Park, et al. (2018) for a similar approach). In order to limit the possibility for Type I errors (i.e., false positives), correlational analyses are restricted to the change in PPS size (i.e., central point) due to administration of testosterone (i.e., testosterone - placebo). No correlational analysis is conducted on the slope of PPS - as this variable did not change due to testosterone - and no correlational analysis is conducted on central point values during placebo or testosterone (only on the difference of these). In line with our prediction and as illustrated in Figure 3, this analysis suggested that participants with higher trait anxiety were particularly 
prone to enlargements of PPS due to administration of testosterone (Pearson correlation; $r=$ $0.55, \mathrm{p}=0.04)$. None of the BANPS personality trait scales significantly correlated with the change in peripersonal space due to testosterone administration, suggesting a specificity to the effect of anxiety on PPS.

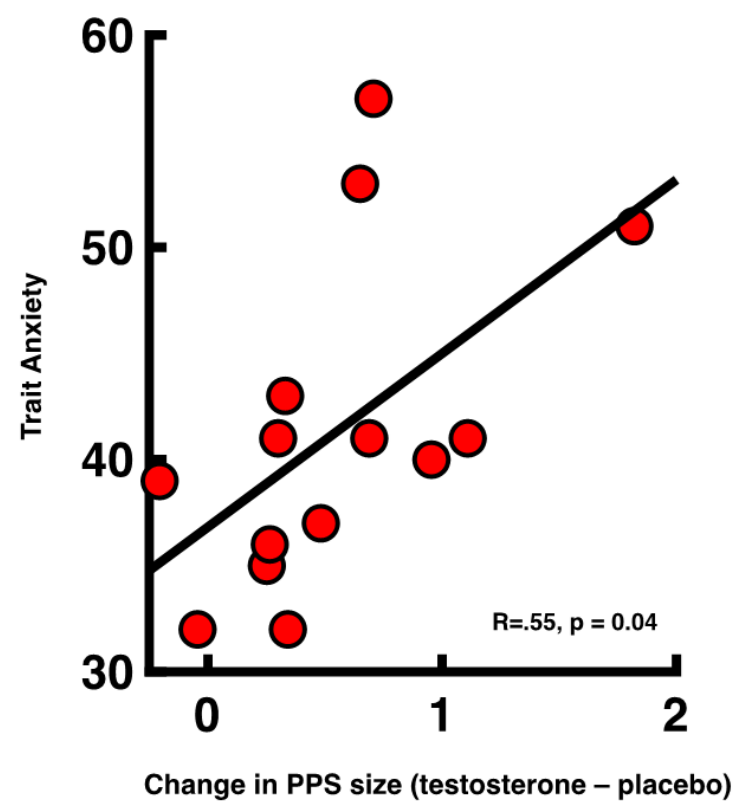

Figure 3. Correlation between trait anxiety and change in self-PPS due to testosterone. Trait anxiety (STAIT) scores (y-axis) as a function of change in PPS size (testosterone - placebo). Each dot represents a participant.

\section{Discussion}

In the present study, we asked whether testosterone facilitates social dominance in part by changing or co-varying with peripersonal space mapping. We used a visuo-tactile interaction task to identify the distance at which an approaching visual stimulus speeded up tactile processing as a proxy for the boundary of PPS. We measured participants' PPS both around their own body and around a confederate unknown to the participant. We measured PPS around the self and other under different levels of testosterone, by means of testosterone and placebo administration. Our results indicated that when testosterone is increased, the PPS around the self expands, while that around the other remains a constant size.

Our finding that testosterone administration caused participants to unconsciously appropriate a larger space as their own suggests that implicit changes in body representation may accompany and even support the well-established effect of testosterone on social dominance 
(for a review see Eisenegger et al. (2011), or Terburg and van Honk (2013)). For instance, previous work found that testosterone administration increased participants' perception of their sensory-motor agency (van der Westhuizen et al., 2017). Furthermore, in the Rubber Hand Illusion, where reductions in limb temperature have been related to a decreased sense of body ownership, testosterone has been shown to prevent this cooling effect (van der Westhuizen, Page, Solms, \& van Honk, 2019). Our current findings extend this evidence by showing that testosterone also changes the encoding of the space immediately surrounding the body, causing an enlargement in participants' PPS. Given that testosterone is known to increase social dominance motivation, the extension of one's own PPS in the presence of another individual in the testosterone condition may be interpreted as an implicit index of increased dominance motivation. This is in keeping with previous research which found that raising testosterone modulated interpersonal distance, causing a significant reduction in the amount of personal distance that healthy male participants preferred from aggressive individuals (Wagels et al., 2017). This finding suggests that the enhanced social aggression associated with testosterone may produce changes in the apportioning of own space. Moreover, a recent study showed that after being primed to feel high power through recollection of a memory in which participants felt powerful, participants' vertical PPS boundary expanded (Vergallito et al., 2019). The authors concluded that high power may cause an expansion of PPS in all directions, and that this should be investigated on the horizontal axis - which is indeed supported by the present study.

In order to better understand the relationship between testosterone and social dominance in terms of PPS representations, we also measured participants' encoding of the PPS around the other (an unknown confederate), with the aim of ascertaining whether testosterone alters selfperception, the perception of others, or both. Our results replicated previous studies showing that PPS can be measured around others (Ishida et al., 2010; Maister et al., 2015; Serino, 2019; Teramoto, 2018) and, importantly, we found that the PPS effect is stronger around the self (represented by a sharper boundary gradient) than around the other. This finding adds to recent evidence suggesting that PPS is the space of the bodily self (Noel et al., 2015; J.-P. Noel et al., 2019; Jean-Paul Noel, Blanke, \& Serino, 2018; Serino, 2019).. However, a limitation in this regard, is that in the VR task used, the virtual ball always travelled toward participants and did not recede in the direction of the confederate. Had the ball approached and receded, the PPS effect around the other may have been stronger, given that neurons encoding for PPS are known to respond preferentially to looming stimuli (Fogassi et al., 1996), and may have been 
represented by a sharper boundary gradient. Our analysis also revealed that the encoding of the PPS boundary of the other was constant across testosterone conditions, suggesting that testosterone alters self-perception rather than other-perception.

Having established a link between raised testosterone and an enlargement in PPS size around the self, we explored whether the increase in PPS size due to testosterone administration may be linked to personality variables. We found a correlation between the increase in PPS size due to testosterone administration and trait anxiety, indicating that participants higher in trait anxiety were most prone to PPS enlargement after testosterone administration. This is noteworthy, given that both anxiety (Iachini, Ruggiero, Ruotolo, di Cola, \& Senese, 2015; Sambo \& Iannetti, 2013; Taffou \& Viaud-Delmon, 2014), and also paradoxically, testosterone, which is known to reduce anxiety (Hermans et al., 2007; Hermans, Putman, Baas, et al., 2006; van Honk et al., 2005), increase PPS. At this point we can only speculate, but it is possible that the enlargement of PPS corresponds to a social coping strategy that anxious individuals employ implicitly to manage feelings of social discomfort and which people with high testosterone utilise instead more proactively as a basic form of empowerment. In this way, as in previous studies (Hermans et al., 2007), the dominance enhancing effects of testosterone may be most pronounced in anxious participants. Future studies that are able to evaluate the emotional and behavioural repercussions of expanded PPS in social settings are needed to disentangle the functional differences in PPS among high and low anxiety groups.

\section{Conclusion}

The present study makes a novel contribution to our understanding of the embodied means by which testosterone enhances social dominance. Our results indicated that raising participants' testosterone caused the PPS around their bodies to expand, while PPS around the confederate remained unaltered. These results suggest that the known relationship between testosterone and the motivation for social dominance may reflect in implicit embodied changes in individuals' sensory-motor processing, and not changes in the perceived sensorimotor affordances of others. Specifically, our findings suggest that the enhanced dominance motivation conferred by testosterone may cause the appropriation of a larger space of the bodily self.

\section{Acknowledgements}

CJM received support from the University of Cape Town and the Ernst \& Ethel Erickson Trust. 


\section{References}

Barrett, F. S., Robins, R. W., \& Janata, P. (2013). A brief form of the Affective Neuroscience Personality Scales. Psychological assessment, 25(3), 826.

Bassolino, M., Finisguerra, A., Canzoneri, E., Serino, A., \& Pozzo, T. (2015). Dissociating effect of upper limb non-use and overuse on space and body representations. Neuropsychologia, 70, 385-392.

Bausenhart, K. M., Di Luca, M., \& Ulrich, R. (2018). Assessing duration discrimination: Psychophysical methods and psychometric function analysis. In Timing and Time Perception: Procedures, Measures, \& Applications (pp. 52-78): Brill.

Bell, M. R., \& Sisk, C. L. (2013). Dopamine mediates testosterone-induced social reward in male Syrian hamsters. Endocrinology, 154(3), 1225-1234.

Bernasconi, F., Noel, J.-P., Park, H., Faivre, N., Seeck, M., Spinelli, L., . . Serino, A. (2018). Audio-tactile and peripersonal space processing around the trunk in human parietal and temporal cortex: an intracranial EEG study. bioRxiv, 249078. doi: $10.1101 / 249078$

Bianchi, V. E. (2019). The anti-inflammatory effects of testosterone. Journal of the Endocrine Society, 3(1), 91-107.

Boksem, M. A., Mehta, P. H., Van den Bergh, B., van Son, V., Trautmann, S. T., Roelofs, K., ... Sanfey, A. G. (2013). Testosterone inhibits trust but promotes reciprocity. Psychological Science, 24(11), 2306-2314.

Bos, P. A., Terburg, D., \& van Honk, J. (2010). Testosterone decreases trust in socially naive humans. Proc Natl Acad Sci U S A, 107(22), 9991-9995. doi:10.1073/pnas.0911700107

Bos, P. A., van Honk, J., Ramsey, N. F., Stein, D. J., \& Hermans, E. J. (2013). Testosterone administration in women increases amygdala responses to fearful and happy faces. Psychoneuroendocrinology, 38(6), 808-817. doi:10.1016/j.psyneuen.2012.09.005

Brozzoli, C., Cardinali, L., Pavani, F., \& Farnè, A. (2010). Action-specific remapping of peripersonal space. Neuropsychologia, 48(3), 796-802. doi:10.1016/j.neuropsychologia.2009.10.009

Canzoneri, E., Ubaldi, S., Rastelli, V., Finisguerra, A., Bassolino, M., \& Serino, A. (2013). Tool-use reshapes the boundaries of body and peripersonal space representations. Experimental Brain Research, 228(1), 25-42. doi:10.1007/s00221-013-3532-2

Davis, K. L., \& Panksepp, J. (2011). The brain's emotional foundations of human personality and the Affective Neuroscience Personality Scales. Neuroscience \& Biobehavioral Reviews, 35(9), 1946-1958.

di Pellegrino, G., \& Làdavas, E. (2015). Peripersonal space in the brain. Neuropsychologia, 66, 126-133. doi:10.1016/j.neuropsychologia.2014.11.011

Duhamel, J.-R., Colby, C. L., \& Goldberg, M. E. (1998). Ventral intraparietal area of the macaque: congruent visual and somatic response properties. Journal of neurophysiology, 79(1), 126-136.

Eisenegger, C., Haushofer, J., \& Fehr, E. (2011). The role of testosterone in social interaction. Trends in Cognitive Sciences, 15(6), 263-271. doi:10.1016/j.tics.2011.04.008

Enter, D., Spinhoven, P., \& Roelofs, K. (2014). Alleviating social avoidance: Effects of single dose testosterone administration on approach-avoidance action. Hormones \& Behavior, 65(4), 351-354. doi:10.1016/j.yhbeh.2014.02.001 
Enter, D., Terburg, D., Harrewijn, A., Spinhoven, P., \& Roelofs, K. (2016). Single dose testosterone administration alleviates gaze avoidance in women with Social Anxiety Disorder. Psychoneuroendocrinology, 63, 26-33. doi:10.1016/j.psyneuen.2015.09.008

Fogassi, L., Gallese, V., Fadiga, L., Luppino, G., Matelli, M., \& Rizzolatti, G. (1996). Coding of peripersonal space in inferior premotor cortex (area F4). Journal of neurophysiology, 76(1), 141-157.

Graziano, M., \& Cooke, D. (2006). Parieto-frontal interactions, personal space, and defensive behavior. Neuropsychologia, 44(13), 2621-2635. doi:10.1016/j.neuropsychologia.2005.09.009

Guterstam, A., Szczotka, J., Zeberg, H., \& Ehrsson, H. H. (2018). Tool use changes the spatial extension of the magnetic touch illusion. Journal of Experimental Psychology: General, 147(2), 298.

Hermans, E. J., Putman, P., Baas, J. M., Gecks, N. M., Kenemans, J. L., \& van Honk, J. (2007). Exogenous testosterone attenuates the integrated central stress response in healthy young women. Psychoneuroendocrinology, 32(8-10), 1052-1061. doi:10.1016/j.psyneuen.2007.08.006

Hermans, E. J., Putman, P., Baas, J. M., Koppeschaar, H. P., \& van Honk, J. (2006). A single administration of testosterone reduces fear-potentiated startle in humans. Biol Psychiatry, 59(9), 872-874. doi:10.1016/j.biopsych.2005.11.015

Hermans, E. J., Putman, P., \& van Honk, J. (2006). Testosterone administration reduces empathetic behavior: a facial mimicry study. Psychoneuroendocrinology, 31(7), 859866. doi:10.1016/j.psyneuen.2006.04.002

Hobeika, L., Taffou, M., Carpentier, T., Warusfel, O., \& Viaud-Delmon, I. (2020). Capturing the dynamics of peripersonal space by integrating expectancy effects and sound propagation properties. Journal of Neuroscience Methods, 332, 108534.

Iachini, T., Coello, Y., Frassinetti, F., Senese, V. P., Galante, F., \& Ruggiero, G. (2016). Peripersonal and interpersonal space in virtual and real environments: Effects of gender and age. Journal of Environmental Psychology, 45, 154-164. doi:https://doi.org/10.1016/i.jenvp.2016.01.004

Iachini, T., Ruggiero, G., Ruotolo, F., di Cola, A. S., \& Senese, V. P. (2015). The influence of anxiety and personality factors on comfort and reachability space: A correlational study. 16(1), 255-258.

Iriki, A., Tanaka, M., \& Iwamura, Y. (1996). Coding of modified body schema during tool use by macaque postcentral neurones. Neuroreport, 7(14), 2325-2330.

Ishida, H., Nakajima, K., Inase, M., \& Murata, A. (2010). Shared Mapping of Own and Others' Bodies in Visuotactile Bimodal Area of Monkey Parietal Cortex. Journal of Cognitive Neuroscience, 22(1), 83-96. doi:10.1162/jocn.2009.21185

Kandula, M., Van der Stoep, N., Hofman, D., \& Dijkerman, H. C. J. E. B. R. (2017). On the contribution of overt tactile expectations to visuo-tactile interactions within the peripersonal space. 235(8), 2511-2522. doi:10.1007/s00221-017-4965-9

Maister, L., Cardini, F., Zamariola, G., Serino, A., \& Tsakiris, M. (2015). Your place or mine: Shared sensory experiences elicit a remapping of peripersonal space. Neuropsychologia, 70, 455-461. doi:10.1016/j.neuropsychologia.2014.10.027

Makin, T. R., Holmes, N. P., \& Ehrsson, H. H. (2008). On the other hand: dummy hands and peripersonal space. Behavioural brain research, 191(1), 1-10.

Mazur, A., \& Booth, A. (1998). Testosterone and dominance in men. Behavioral \& Brain Sciences, 21(3), 353. doi:10.1017/S0140525X98001228

Miller, J., \& Ulrich, R. (2001). On the analysis of psychometric functions: The SpearmanKärber method. Perception \& Psychophysics, 63(8), 1399-1420. 
Montoya, E. R., Terburg, D., Bos, P. A., Will, G.-J., Buskens, V., Raub, W., \& van Honk, J. (2013). Testosterone administration modulates moral judgments depending on second-to-fourth digit ratio. Psychoneuroendocrinology, 38(8), 1362-1369.

Noel, Pfeiffer, C., Blanke, O., \& Serino, A. (2015). Peripersonal space as the space of the bodily self. Cognition, 144, 49-57. doi:10.1016/j.cognition.2015.07.012

Noel, J.-P., Blanke, O., Magosso, E., \& Serino, A. (2018). Neural adaptation accounts for the dynamic resizing of peripersonal space: evidence from a psychophysicalcomputational approach. 119(6), 2307-2333. doi:10.1152/jn.00652.2017

Noel, J.-P., Chatelle, C., Perdikis, S., Jöhr, J., Da Silva, M. L., Ryvlin, P., . . Serino, A. (2019). Peri-personal space encoding in patients with disorders of consciousness and cognitive-motor dissociation. NeuroImage: Clinical, 24, 101940.

Noel, J.-P., Grivaz, P., Marmaroli, P., Lissek, H., Blanke, O., \& Serino, A. (2015). Full body action remapping of peripersonal space: The case of walking. Neuropsychologia, 70, 375-384. doi:10.1016/j.neuropsychologia.2014.08.030

Noel, J.-P., Park, H.-D., Pasqualini, I., Lissek, H., Wallace, M., Blanke, O., \& Serino, A. (2018). Audio-visual sensory deprivation degrades visuo-tactile peri-personal space. Consciousness and Cognition, 61, 61-75. doi:https://doi.org/10.1016/i.concog.2018.04.001

Noel, J. P., Bertoni, T., Terrebonne, E., Pellencin, E., Herbelin, B., Cascio, C., . . . Serino, A. (2020). Rapid Recalibration of Peri-Personal Space: Psychophysical, Electrophysiological, and Neural Network Modeling Evidence. Cereb Cortex. doi:10.1093/cercor/bhaa103

Noel, J. P., Blanke, O., \& Serino, A. (2018). From multisensory integration in peripersonal space to bodily self-consciousness: from statistical regularities to statistical inference. Annals of the New York Academy of Sciences, 1426(1), 146-165. doi:10.1111/nyas.13867

Noel, J. P., Samad, M., Doxon, A., Clark, J., Keller, S., \& Di Luca, M. (2018). Peri-personal space as a prior in coupling visual and proprioceptive signals. Sci Rep, 8(1), 15819. doi:10.1038/s41598-018-33961-3

Patané, I., Cardinali, L., Salemme, R., Pavani, F., Farnè, A., \& Brozzoli, C. (2019). Action planning modulates peripersonal space. Journal of Cognitive Neuroscience, 31(8), 1141-1154.

Pellencin, E., Paladino, M. P., Herbelin, B., \& Serino, A. (2018). Social perception of others shapes one's own multisensory peripersonal space. Cortex, 104, 163-179. doi:https://doi.org/10.1016/j.cortex.2017.08.033

Pfeiffer, C., Noel, J.-P., Serino, A., \& Blanke, O. (2018). Vestibular modulation of peripersonal space boundaries. 47(7), 800-811. doi:doi:10.1111/ejn.13872

Rizzolatti, G., Scandolara, C., Matelli, M., \& Gentilucci, M. (1981a). Afferent properties of periarcuate neurons in macaque monkeys. I. Somatosensory responses. Behavioural brain research, 2(2), 125-146.

Rizzolatti, G., Scandolara, C., Matelli, M., \& Gentilucci, M. (1981b). Afferent properties of periarcuate neurons in macaque monkeys. II. Visual responses. Behavioural brain research, 2(2), 147-163.

Salomon, R., Noel, J.-P., Łukowska, M., Faivre, N., Metzinger, T., Serino, A., \& Blanke, O. (2017). Unconscious integration of multisensory bodily inputs in the peripersonal space shapes bodily self-consciousness. Cognition, 166, 174-183.

doi:https://doi.org/10.1016/j.cognition.2017.05.028

Sambo, C. F., \& Iannetti, G. D. (2013). Better Safe Than Sorry? The Safety Margin Surrounding the Body Is Increased by Anxiety. Journal of Neuroscience, 33(35), 14225-14230. doi:10.1523/JNEUROSCI.0706-13.2013 
Schutter, D. J., \& Honk, J. v. (2004). Decoupling of midfrontal delta-beta oscillations after testosterone administration. International Journal of Psychophysiology, 53, 71-73.

Serino, A. (2019). Peripersonal space (PPS) as a multisensory interface between the individual and the environment, defining the space of the self. Neuroscience \& Biobehavioral Reviews. doi:https://doi.org/10.1016/j.neubiorev.2019.01.016

Serino, A., Bassolino, M., Farne, A., \& Ladavas, E. J. P. s. (2007). Extended multisensory space in blind cane users. 18(7), 642-648.

Serino, A., Canzoneri, E., Marzolla, M., di Pellegrino, G., \& Magosso, E. (2015). Extending peripersonal space representation without tool-use: evidence from a combined behavioral-computational approach. 9(4). doi:10.3389/fnbeh.2015.00004

Serino, A., Noel, J.-P., Galli, G., Canzoneri, E., Marmaroli, P., Lissek, H., \& Blanke, O. J. S. r. (2015). Body part-centered and full body-centered peripersonal space representations. $5,18603$.

Serino, A., Noel, J.-P., Mange, R., Canzoneri, E., Pellencin, E., Ruiz, J. B., . . Herbelin, B. (2018). Peripersonal Space: An Index of Multisensory Body-Environment Interactions in Real, Virtual, and Mixed Realities. 4(31). doi:10.3389/fict.2017.00031

Sinervo, B., Miles, D. B., Frankino, W. A., Klukowski, M., \& DeNardo, D. F. (2000). Testosterone, endurance, and Darwinian fitness: natural and sexual selection on the physiological bases of alternative male behaviors in side-blotched lizards. Hormones and Behavior, 38(4), 222-233.

Sinnesael, M., Boonen, S., Claessens, F., Gielen, E., \& Vanderschueren, D. (2011). Testosterone and the male skeleton: a dual mode of action. Journal of osteoporosis, 2011.

Stanton, S. J., \& Schultheiss, O. C. (2009). The hormonal correlates of implicit power motivation. Journal of Research in Personality, 43(5), 942-949. doi:10.1016/j.jrp.2009.04.001

Stone, K. D., Kandula, M., Keizer, A., \& Dijkerman, H. C. (2018). Peripersonal space boundaries around the lower limbs. Experimental Brain Research, 236(1), 161-173.

Taffou, M., \& Viaud-Delmon, I. J. F. i. p. (2014). Cynophobic fear adaptively extends peripersonal space. $5,122$.

Teneggi, C., Canzoneri, E., di Pellegrino, G., \& Serino, A. (2013). Social Modulation of Peripersonal Space Boundaries. Current Biology, 23(5), 406-411. doi:10.1016/j.cub.2013.01.043

Teramoto, W. (2018). A behavioral approach to shared mapping of peripersonal space between oneself and others. Scientific Reports, 8(1), 5432. doi:10.1038/s41598-01823815-3

Terburg, D., Aarts, H., \& van Honk, J. (2012). Testosterone Affects Gaze Aversion From Angry Faces Outside of Conscious Awareness. Psychological Science (0956-7976), 23(5), 459-463. doi:10.1177/0956797611433336

Terburg, D., Syal, S., Rosenberger, L. A., Heany, S. J., Stein, D. J., \& Honk, J. v. (2016). Testosterone abolishes implicit subordination in social anxiety. Psychoneuroendocrinology, 72, 205-211. doi:10.1016/j.psyneuen.2016.07.203

Terburg, D., \& van Honk, J. (2013). Approach-Avoidance versus DominanceSubmissiveness: A Multilevel Neural Framework on How Testosterone Promotes Social Status. 5(3), 296-302. doi:10.1177/1754073913477510

Tuiten, A., Van Honk, J., Koppeschaar, H., Bernaards, C., Thijssen, J., \& Verbaten, R. (2000). Time Course of Effects of Testosterone Administration on Sexual Arousal in Women. Archives of General Psychiatry, 57(2), 149-153. doi:10.1001/archpsyc.57.2.149 
van der Westhuizen, D., Moore, J., Solms, M., \& van Honk, J. (2017). Testosterone facilitates the sense of agency. Consciousness \& Cognition, 56, 58-67. doi:10.1016/j.concog.2017.10.005

van der Westhuizen, D., Page, T., Solms, M., \& van Honk, J. (2019). The territory of my body: Testosterone prevents limb cooling in the Rubber Hand Illusion. Multisensory research, 1(aop), 1-27.

van der Westhuizen, D., \& Solms, M. (2015). Social dominance and the Affective Neuroscience Personality Scales. Consciousness and Cognition, 33, 90-111. doi:https://doi.org/10.1016/j.concog.2014.12.005

van Honk, J., Peper, J. S., \& Schutter, D. J. L. G. (2005). Testosterone Reduces Unconscious Fear but Not Consciously Experienced Anxiety: Implications for the Disorders of Fear and Anxiety. Biological Psychiatry, 58(3), 218-225. doi:https://doi.org/10.1016/j.biopsych.2005.04.003

van Honk, J., \& Schutter, D. J. (2007). Testosterone reduces conscious detection of signals serving social correction: implications for antisocial behavior. Psychol Sci, 18(8), 663-667. doi:10.1111/j.1467-9280.2007.01955.x

van Honk, J., Schutter, D. J., Bos, P. A., Kruijt, A.-W., Lentjes, E. G., \& Baron-Cohen, S. (2011). Testosterone administration impairs cognitive empathy in women depending on second-to-fourth digit ratio. Proceedings of the National Academy of Sciences, 201011891. doi:10.1073/pnas.1011891108

van Honk, J., \& Tuiten, A. (2001). A Single Administration of Testosterone Induces Cardiac Accelerative Responses to Angry Faces in Healthy Young Women. Behavioral Neuroscience, 115(1), 238. doi:10.1037/0735-7044.115.1.238

van Honk, J., Tuiten, A., Hermans, E., Putman, P., Koppeschaar, H., Thijssen, J., . . van Doornen, L. (2001). A single administration of testosterone induces cardiac accelerative responses to angry faces in healthy young women. Behav Neurosci, 115(1), 238-242. doi:10.1037/0735-7044.115.1.238

van Honk, J., Tuiten, A., Verbaten, R., van den Hout, M., Koppeschaar, H., Thijssen, J., \& de Haan, E. (1999). Correlations among Salivary Testosterone, Mood, and Selective Attention to Threat in Humans. Hormones and Behavior, 36(1), 17-24. doi:https://doi.org/10.1006/hbeh.1999.1521

Vergallito, A., Gerfo, E. L., Varoli, E., Brambilla, M., Sacchi, S., Anzani, S., \& Lauro, L. R. (2019). Positive self-perception and corticospinal excitability: Recalling positive behavior expands peripersonal space boundaries. Neuropsychologia, 135, 107224.

Wagels, L., Radke, S., Goerlich, K. S., Habel, U., \& Votinov, M. (2017). Exogenous testosterone decreases men's personal distance in a social threat context. Hormones \& Behavior, 90, 75-83. doi:10.1016/j.yhbeh.2017.03.001

Wang, C., Swerdloff, R. S., Iranmanesh, A., Dobs, A., Snyder, P. J., Cunningham, G., . . . Berman the Testosterone Gel Study Group, N. (2000). Transdermal testosterone gel improves sexual function, mood, muscle strength, and body composition parameters in hypogonadal men. The Journal of Clinical Endocrinology \& Metabolism, 85(8), 2839-2853.

Wirth, M. M., \& Schultheiss, O. C. (2007). Basal testosterone moderates responses to anger faces in humans. Physiology \& Behavior, 90(2), 496-505. doi:https://doi.org/10.1016/j.physbeh.2006.10.016

Wright, N. D., Bahrami, B., Johnson, E., Malta, G. D., Rees, G., Frith, C. D., \& Dolan, R. J. (2012). Testosterone disrupts human collaboration by increasing egocentric choices. 279(1736), 2275-2280. doi:doi:10.1098/rspb.2011.2523 